\title{
Performance Improvement of Robust Controllers for Polynomially Uncertain Systems
}

\author{
Javad Lavaei ${ }^{\mathrm{a}}$, Amir G. Aghdam ${ }^{\mathrm{b}}$ \\ ${ }^{a}$ Department of Control and Dynamical Systems, California Institute of Technology, Pasadena, CA 91125, USA \\ ${ }^{\mathrm{b}}$ Department of Electrical and Computer Engineering, Concordia University, Montréal, QC H3G 1M8, Canada
}

\begin{abstract}
This paper is concerned with the high-performance robust control of discrete-time linear time-invariant (LTI) systems with semi-algebraic uncertainty regions. It is assumed that a robustly stabilizing static controller is given whose gain depends polynomially on the uncertain variables. The problem of tuning this parameter-dependent gain with respect to a prescribed quadratic cost function is formulated as a sum-of-squares (SOS) optimization. This method leads to a near-optimal controller whose performance is better than that of the initial controller. It is shown that the results derived in the present work encompass the ones obtained in a recent paper. The efficacy of the results is elucidated by an example.
\end{abstract}

Key words: Optimal controller synthesis for systems with uncertainties, robust control, convex optimization.

\section{Introduction}

It is known that all physical systems are subject to uncertainty or parameter perturbation. Robustness analysis for different classes of uncertain control systems has been extensively studied in the literature (Sekaj and Vesely, 2005; Bakhilina and Stepanov, 2001; Lin, Wang and Lee, 2006; Feron, Apkarian and Gahinet, 1996). Robust stability verification can be envisaged as one of the most important problems in this area. This problem is concerned with the conditions under which a controller designed for a nominal model can also stabilize the corresponding uncertain system. Robust stability verification has been addressed in the literature for different types of uncertainties (e.g., structured and unstructured (Foo and Soh, 1993)) in the past several years. More recently, the important class of parametric uncertainty has drawn much attention in this field.

Presently, the most efficient technique for verifying the robust stability of a system under a nominal controller is to check the existence of a proper Lyapunov function for the closed-loop system (Chesi, Garulli, Tesi and

* This work has been supported by the Natural Sciences and Engineering Research Council of Canada under grant RGPIN-262127-07.

Email addresses: lavaei@cds.caltech.edu (Javad Lavaei), aghdam@ece.concordia.ca (Amir G. Aghdam).
Vicino, 2005; Lavaei and Aghdam, 2006a; Oliveira and Peres, 2006). For the sake of computational simplicity, the (Chesi, Garulli, Tesi and Vicino, 2005; Lavaei and Aghdam, 2006a; Oliveira and Peres, 2006). For the sake of computational simplicity, the pioneer works sought a fixed Lyapunov function. While this method may work satisfactorily for some systems, it is known that the corresponding robust stability results can be quite conservative in general. As an alternative, many of the recent works consider parameter-dependent Lyapunov functions in order to achieve less conservative results. Notice that different types of Lyapunov functions can be considered here, e.g. sinusoidal, exponential, etc. Nonetheless, it is shown in Bliman (2004) that the polynomial-type Lyapunov functions are always capable of detecting the robust stability of any robustly stable system.

As the simplest scenario, assume that the region of uncertainty is polytopic. The works Oliveira and Geromel (2005) and Kau, Liu, Hong, Lee, Fang and Lee (2005) search for a Lyapunov function in the form of a firstorder polynomial to determine the robust stability of the system. These works present relatively simple linear matrix inequalities (LMI) as sufficient conditions for stability, which are proved to be very conservative in various examples. As a more sophisticated but less conservative approach, it is shown in Chesi, Garulli, Tesi and Vicino (2005) that robust stability over a polytope 
is tantamount to the existence of a Lyapunov function in the form of a homogeneous polynomial with a certain bound on its degree. Hence, the seminal work Chesi, Garulli, Tesi and Vicino (2005) properly characterizes all the essential candidates for the desired Lyapunov function. A sufficient LMI condition is subsequently derived in Chesi, Garulli, Tesi and Vicino (2005) to check the robust stability of the system. A method similar to Chesi, Garulli, Tesi and Vicino (2005) is also proposed in Oliveira and Peres (2006), which seeks the same type of Lyapunov function. Nevertheless, the work Oliveira and Peres (2006) further simplifies the LMI conditions at the cost of introducing more conservatism.

More recently, it is asserted in Lavaei and Aghdam (2008) that the robust stability verification of a system over any semi-algebraic set satisfying a mild condition is equivalent to checking the existence of a set of polynomials for which a specific sum-of-squares (SOS) matrix equation holds. The feasibility of this matrix equation can be determined systematically as long as some bounds on the degrees of the above-mentioned polynomials are known a priori. The work Lavaei and Aghdam (2008) also presents important results on how to compute these bounds. It is worth mentioning that the conditions obtained in Lavaei and Aghdam (2008) encompass those derived in both Chesi, Garulli, Tesi and Vicino (2005) and Oliveira and Peres (2006) for the particular case of polytopic uncertainty.

In addition to the surveyed papers dealing with the robust stability problem, there have been some other works concerned with the robust control synthesis and robust performance analysis (Vesely, 2005; Gao, Lam, Xie and Wang, 2005; Oliveira, Oliveira, Leite, Montagner and Peres, 2004; Goncalves, Palhares and Takahashi, 2005; Shaked, 2003). The latter problem (robust synthesis) is known to be more sophisticated than the former one (robust stability), and the available design techniques are not satisfactory in all respects. For instance, a method is proposed in Vesely (2005) to design a near-optimal controller for systems with a polytopic uncertainty. However, due to the complexity of the problem, the controller presented in the above work may perform poorly in general, as it relies on some sufficient LMI conditions. There are some other results in the literature dealing with $\mathrm{H}_{2}$ or $H_{\infty}$ robust controller design, which normally suffer from the same weak points, e.g. see Gao, Lam, Xie and Wang (2005); Shaked (2003).

This work deals with uncertain linear time-invariant (LTI) discrete-time systems whose uncertainty belongs to a semi-algebraic set. It is assumed that a robustly stabilizing static controller is initially available, whose gain can depend polynomially on the uncertain variables. The objective is to tune the gain of this controller so that its performance is improved with respect to a prescribed quadratic performance index. To this end, it is shown that the closed-loop Lyapunov matrix is a rational function, which can be approximated by a polynomial matrix satisfying an elegant inequality. An upper bound is also derived in order to determine the accuracy of this approximation. An SOS method is then developed to solve the underlying problem. It is worth noting that the idea of designing a parameter-dependent static controller has also been used in a number of other papers; for instance, see Gao, Lam, Xie and Wang (2005) for the filtering application. The results obtained here are shown to encompass the ones presented in Vesely (2005).

This paper is organized as follows. The problem is formulated in Section 2, where some definitions and convenient notations are also introduced. The main results are provided in Section 3, followed by an illustrative example in Section 4. Finally, some concluding remarks are drawn in Section 5.

\section{Problem formulation}

Consider an uncertain discrete-time system $\mathcal{S}(\boldsymbol{\alpha})$ with the state-space representation:

$$
\begin{aligned}
x[\kappa+1] & =A(\boldsymbol{\alpha}) x[\kappa]+B(\boldsymbol{\alpha}) u[\kappa] \\
y[\kappa] & =C(\boldsymbol{\alpha}) x[\kappa], \quad \kappa \in \mathbb{Z}
\end{aligned}
$$

where:

- $x[\kappa] \in \Re^{n}, u[\kappa] \in \Re^{m}$ and $y[\kappa] \in \Re^{r}$ are the state, input and output of $\mathcal{S}(\boldsymbol{\alpha})$, respectively.

- The vector $\boldsymbol{\alpha}=\left[\begin{array}{llll}\alpha_{1} & \alpha_{2} & \cdots & \alpha_{\mu}\end{array}\right]$ denotes the uncertain parameters of the system.

- $A(\boldsymbol{\alpha}), B(\boldsymbol{\alpha})$ and $C(\boldsymbol{\alpha})$ are matrix polynomials in terms of the variable $\boldsymbol{\alpha}$, and have appropriate dimensions.

Assume that the uncertainty region $\mathcal{D}$ associated with the system $\mathcal{S}(\boldsymbol{\alpha})$ is a semi-algebraic set defined as follows:

$$
\mathcal{D}:=\left\{\boldsymbol{\alpha} \mid q_{1}(\boldsymbol{\alpha}) \geq 0, \ldots, q_{\eta}(\boldsymbol{\alpha}) \geq 0\right\}
$$

where $q_{1}(\boldsymbol{\alpha}), q_{2}(\boldsymbol{\alpha}), \ldots, q_{\eta}(\boldsymbol{\alpha})$ are given scalar polynomials. Assume also that the initial state of the system is a random variable with zero mean and the co-variance matrix $X_{0}$, i.e., $\mathcal{E}\left\{x(0) x(0)^{T}\right\}=X_{0}$ (note that $\mathcal{E}\{\cdot\}$ represents the expectation operator).

Definition 1 For every static LTI controller $\mathbf{K}$ and uncertainty vector $\boldsymbol{\alpha} \in \mathcal{D}$, define the performance index:

$$
J(\boldsymbol{\alpha}, \mathbf{K})=\mathcal{E}\left\{\sum_{\kappa=0}^{\infty}\left(\tilde{x}[\kappa]^{T} Q(\boldsymbol{\alpha}) \tilde{x}[\kappa]+\tilde{u}[\kappa]^{T} R \tilde{u}[\kappa]\right)\right\}
$$

where: 
- $\tilde{x}[\kappa]$ and $\tilde{u}[\kappa]$ denote the state and input of the resultant closed-loop system, respectively.

- $R$ is a fixed positive definite matrix.

- $Q(\boldsymbol{\alpha})$ is a matrix polynomial that is positive definite over the region $\mathcal{D}$.

Notice that the performance index $J(\boldsymbol{\alpha}, \mathbf{K})$ is defined in such a way that it penalizes the input of the system by a constant term $R$, but balances the state of the system by a parameter dependent (non-fixed) term $Q(\boldsymbol{\alpha})$.

Definition 2 Given the system $\mathcal{S}(\boldsymbol{\alpha})$ and a controller $\mathbf{K}$, define a performance index for the closed-loop system over the entire uncertainty region $\mathcal{D}$ as follows:

$$
J(\mathbf{K})=\int_{\mathcal{D}} J(\boldsymbol{\alpha}, \mathbf{K}) f(\boldsymbol{\alpha}) d \boldsymbol{\alpha}
$$

where $f(\boldsymbol{\alpha})$ is a given weighting function specifying the relative importance of the performance of the system at different points in the uncertainty region $\boldsymbol{\alpha} \in \mathcal{D}$.

Assume that initially a static parameter-dependent controller $\mathbf{K}_{0}$ is given, which robustly stabilizes the system $\mathcal{S}$ over the uncertainty region $\mathcal{D}$. Let this controller be represented by the control law $u[\kappa]=K_{0}(\boldsymbol{\alpha}) y[\kappa]$, where $K_{0}(\boldsymbol{\alpha})$ is a matrix polynomial of degree $n_{0}$. Note that the robust stability of a control system can be checked using the existing techniques such as the one given in Lavaei and Aghdam (2008). The objective is to tune the coefficients of the polynomial $K_{0}(\boldsymbol{\alpha})$ in such a way that the performance index $J(\mathbf{K})$ is reduced in some sense. More precisely, the purpose of this work is to design a near-optimal parameter-dependent static controller, using $\mathbf{K}_{0}$ as the initial point in the optimization procedure.

The following definitions and assumptions are needed for the development of the main results.

Definition 3 A matrix polynomial $C(\boldsymbol{\alpha})$ is said to be sum-of-squares (SOS) if there exists a matrix polynomial $E(\boldsymbol{\alpha})$ such that:

$$
C(\boldsymbol{\alpha})=E^{T}(\boldsymbol{\alpha}) E(\boldsymbol{\alpha})
$$

Definition 4 For every matrix $W$, define vec $\{W\}$ to be a column vector obtained from $W$ by placing its columns under each other successively. For example, $\operatorname{vec}\{\operatorname{eye}(2)\}=\left[\begin{array}{llll}1 & 0 & 0 & 1\end{array}\right]^{T}$.

Notation 1 For the sake of simplicity, the upper block entries of a symmetric block matrix will be displayed by the symbol "*" throughout the paper.

Assumption 1 The set $\mathcal{D}$ is compact and there exist SOS scalar polynomials $w_{0}(\boldsymbol{\alpha}), w_{1}(\boldsymbol{\alpha}), \ldots, w_{\eta}(\boldsymbol{\alpha})$, such that the set of all vectors $\boldsymbol{\alpha}$ satisfying the inequality:

$$
w_{0}(\boldsymbol{\alpha})+w_{1}(\boldsymbol{\alpha}) q_{1}(\boldsymbol{\alpha})+\cdots+w_{\eta}(\boldsymbol{\alpha}) q_{\eta}(\boldsymbol{\alpha}) \geq 0
$$

is compact, where $q_{i}(\boldsymbol{\alpha}), i=1,2, \ldots, \eta$, are given in (2).

\section{Near-optimal robust controller}

The problem of designing a robustly stabilizing controller $\mathbf{K}_{k}$ that outperforms the initial controller (i.e. $\left.J\left(\mathbf{K}_{k}\right)<J\left(\mathbf{K}_{0}\right)\right)$ will be addressed in the sequel.

Lemma 1 Assume that a controller $\mathbf{K}$ with the control law $u[\kappa]=K(\boldsymbol{\alpha}) y[\kappa]$ stabilizes the uncertain system $\mathcal{S}(\boldsymbol{\alpha})$ over the region $\mathcal{D}$. Then, the performance index $J(\mathbf{K})$ can be obtained as follows:

$$
J(\mathbf{K})=\operatorname{trace}\left(X_{0} \int_{\mathcal{D}} G(\boldsymbol{\alpha}) f(\boldsymbol{\alpha}) d \boldsymbol{\alpha}\right)
$$

where $G(\boldsymbol{\alpha})$ is the solution of the discrete Lyapunov equation given below:

$$
\begin{aligned}
& (A(\boldsymbol{\alpha})+B(\boldsymbol{\alpha}) K(\boldsymbol{\alpha}) C(\boldsymbol{\alpha}))^{T} G(\boldsymbol{\alpha})(A(\boldsymbol{\alpha})+ \\
& B(\boldsymbol{\alpha}) K(\boldsymbol{\alpha}) C(\boldsymbol{\alpha}))-G(\boldsymbol{\alpha})+Q(\boldsymbol{\alpha}) \\
& +C(\boldsymbol{\alpha})^{T} K(\boldsymbol{\alpha})^{T} R K(\boldsymbol{\alpha}) C(\boldsymbol{\alpha})=0
\end{aligned}
$$

Proof: It is well-known that $J(\boldsymbol{\alpha}, \mathbf{K})$ can be expressed as trace $\left(X_{0} G(\boldsymbol{\alpha})\right)$, where $G(\boldsymbol{\alpha})$ satisfies the discrete Lyapunov equation (8) (see Lavaei and Aghdam (2007a) for more details). Thus, one can write:

$$
\begin{aligned}
J(\mathbf{K}) & =\int_{\mathcal{D}} J(\boldsymbol{\alpha}, \mathbf{K}) f(\boldsymbol{\alpha}) d \boldsymbol{\alpha} \\
& =\operatorname{trace}\left(X_{0} \int_{\mathcal{D}} G(\boldsymbol{\alpha}) f(\boldsymbol{\alpha}) d \boldsymbol{\alpha}\right)
\end{aligned}
$$

This completes the proof.

Lemma 2 Assume that the system $\mathcal{S}(\boldsymbol{\alpha})$ is robustly stable under a static controller $\mathbf{K}$ with the control law $u[\kappa]=$ $K(\boldsymbol{\alpha}) y[\kappa]$. There exist a matrix polynomial $H(\boldsymbol{\alpha})$ and a scalar polynomial $h(\boldsymbol{\alpha})$ such that the matrix $G(\boldsymbol{\alpha})$ satisfying the equation (8) can be written as $G(\boldsymbol{\alpha})=\frac{H(\boldsymbol{\alpha})}{h(\boldsymbol{\alpha})}$, where both of the polynomials $H(\boldsymbol{\alpha})$ and $h(\boldsymbol{\alpha})$ are positive definite over the region $\mathcal{D}$.

Proof: The matrix equation (8) can be solved for $G(\boldsymbol{\alpha})$ using the Kronecker product technique as follows:

$$
\begin{aligned}
\operatorname{vec}\{G(\boldsymbol{\alpha})\}= & M(\boldsymbol{\alpha})^{-1} \operatorname{vec}\{Q(\boldsymbol{\alpha}) \\
& \left.+C(\boldsymbol{\alpha})^{T} K(\boldsymbol{\alpha})^{T} R K(\boldsymbol{\alpha}) C(\boldsymbol{\alpha})\right\}
\end{aligned}
$$


where:

$$
\begin{aligned}
M(\boldsymbol{\alpha})=-(A(\boldsymbol{\alpha}) & +B(\boldsymbol{\alpha}) K(\boldsymbol{\alpha}) C(\boldsymbol{\alpha}))^{T} \otimes(A(\boldsymbol{\alpha}) \\
& +B(\boldsymbol{\alpha}) K(\boldsymbol{\alpha}) C(\boldsymbol{\alpha}))^{T}+I
\end{aligned}
$$

(the symbol $\otimes$ stands for the Kronecker product). Define now:

$$
h(\boldsymbol{\alpha})=\operatorname{det}(M(\boldsymbol{\alpha})), \quad H(\boldsymbol{\alpha})=\operatorname{det}(M(\boldsymbol{\alpha})) G(\boldsymbol{\alpha})
$$

It remains to show that:

i) $h(\boldsymbol{\alpha})$ and $H(\boldsymbol{\alpha})$ are both (matrix) polynomials.

ii) $h(\boldsymbol{\alpha})$ and $H(\boldsymbol{\alpha})$ are both positive definite over the region $\mathcal{D}$.

To prove property (i), notice that since $M(\boldsymbol{\alpha})$ is a matrix polynomial, its determinant is also a (scalar) polynomial. Hence, $h(\boldsymbol{\alpha})$ is a polynomial, and so is $H(\boldsymbol{\alpha})$ (in light of equation (10) and (12)). To show the validity of property (ii), denote the eigenvalues of $A(\boldsymbol{\alpha})+B(\boldsymbol{\alpha}) K(\boldsymbol{\alpha}) C(\boldsymbol{\alpha})$ with $\lambda_{1}(\boldsymbol{\alpha}), \lambda_{2}(\boldsymbol{\alpha}), \ldots, \lambda_{n}(\boldsymbol{\alpha})$. One can write:

$$
h(\boldsymbol{\alpha})=\prod_{i=1}^{n} \prod_{j=1}^{n}\left(1-\lambda_{i}(\boldsymbol{\alpha}) \lambda_{j}(\boldsymbol{\alpha})\right)
$$

Since $A(\boldsymbol{\alpha})+B(\boldsymbol{\alpha}) K(\boldsymbol{\alpha}) C(\boldsymbol{\alpha})$ is robustly stable, all eigenvalues $\lambda_{1}(\boldsymbol{\alpha}), \ldots, \lambda_{n}(\boldsymbol{\alpha})$ are inside the unit circle. It follows from this fact and the above equation that $h(\boldsymbol{\alpha})$ is positive over the region $\mathcal{D}$. On the other hand, the robust stability of the closed-loop system implies that the matrix $G(\boldsymbol{\alpha})$ is positive definite over the uncertainty region. As a result, it follows from (12) that $H(\boldsymbol{\alpha})$ is also positive definite over the region $\mathcal{D}$.

Definition 5 Given a positive integeri and a parameterdependent static controller $\mathbf{K}$ with the polynomial gain $K(\boldsymbol{\alpha})$, consider an optimization problem whose objective is to minimize the function:

$$
\operatorname{trace}\left(X_{0} \int_{\mathcal{D}} P(\boldsymbol{\alpha}) f(\boldsymbol{\alpha}) d \boldsymbol{\alpha}\right)
$$

subject to the following constraint:

$$
\Gamma(\boldsymbol{\alpha}):=\left[\begin{array}{ccc}
\Gamma_{11}(\boldsymbol{\alpha}) & * & * \\
\Gamma_{21}(\boldsymbol{\alpha}) & \Gamma_{22}(\boldsymbol{\alpha}) & * \\
\Gamma_{31}(\boldsymbol{\alpha}) & \Gamma_{32}(\boldsymbol{\alpha}) & -I
\end{array}\right]<0, \quad \forall \boldsymbol{\alpha} \in \mathcal{D}
$$

with the (symmetric polynomial matrix) variable $P(\boldsymbol{\alpha}) \in$ $\Re^{n \times n}$ of degree $i$, where:

$$
\begin{aligned}
& \Gamma_{11}(\boldsymbol{\alpha})=-P(\boldsymbol{\alpha})+Q(\boldsymbol{\alpha}), \Gamma_{21}(\boldsymbol{\alpha})=P(\boldsymbol{\alpha}) A(\boldsymbol{\alpha}), \\
& \Gamma_{22}(\boldsymbol{\alpha})=-P(\boldsymbol{\alpha})-P(\boldsymbol{\alpha}) B(\boldsymbol{\alpha}) R^{-1} B(\boldsymbol{\alpha})^{T} P(\boldsymbol{\alpha}), \\
& \Gamma_{31}(\boldsymbol{\alpha})=R^{\frac{1}{2}} K(\boldsymbol{\alpha}) C(\boldsymbol{\alpha}), \Gamma_{32}(\boldsymbol{\alpha})=R^{-\frac{1}{2}} B(\boldsymbol{\alpha})^{T} P(\boldsymbol{\alpha})
\end{aligned}
$$

Denote the infimum of this minimization problem with $J_{i}(\mathbf{K})$.

Theorem 1 Consider a static controller $\mathbf{K}$ with the parameter-dependent gain $K(\boldsymbol{\alpha})$. Then, the following statements are true:

i) The infinite sequence $J_{1}(\mathbf{K}), J_{2}(\mathbf{K}), J_{3}(\mathbf{K}), \ldots$ is nonincreasing.

ii) $J_{i}(\mathbf{K})$ is always greater than or equal to $J(\mathbf{K})$, for every natural number $i$.

Proof: The proof is straightforward. The details are omitted for brevity.

In the case when the system $\mathcal{S}(\boldsymbol{\alpha})$ is not uncertain and the matrix polynomials of the system are all of degree 0 , it can be inferred from Cao and Lam (2001) that the relation $J_{i}(\mathbf{K})=J(\mathbf{K})$ holds for all positive integers $i$. In the general case, however, $J(\mathbf{K})$ is obtained by solving an integral involving a rational function (i.e. $G(\boldsymbol{\alpha})$ ), whereas $J_{i}\left(\mathbf{K}_{k}\right)$ is obtained by solving the same integral after substituting the above-mentioned rational function with a proper polynomial (i.e. $P(\boldsymbol{\alpha})$ ). This implies that the equality $J_{i}(\mathbf{K})=J(\mathbf{K})$ may not be achieved for an uncertain system even if the index $i$ is arbitrarily large. This problem will be addressed in the sequel.

Consider $h(\boldsymbol{\alpha})$ and $H(\boldsymbol{\alpha})$ introduced in Lemma 2. It results from the compactness of $\mathcal{D}$ that there exist two positive numbers $\mu_{1}$ and $\mu_{2}$ with the property:

$$
0<\mu_{1} \leq h(\boldsymbol{\alpha}) \leq \mu_{2}, \quad \forall \boldsymbol{\alpha} \in \mathcal{D}
$$

Define $\rho(i):=(2 i-1) \operatorname{deg}(h)+\operatorname{deg}(H)$, for every natural number $i$.

Theorem 2 Assume that the system $\mathcal{S}(\boldsymbol{\alpha})$ is robustly stable under a static controller $\mathbf{K}$ with the control law $u[\kappa]=K(\boldsymbol{\alpha}) y[\kappa]$. The infinite subsequence $\left\{J_{\rho(i)}(\mathbf{K})\right\}_{1}^{\infty}$ of the sequence $\left\{J_{i}(\mathbf{K})\right\}_{1}^{\infty}$ converges exponentially to $J(\mathbf{K})$ from above, and also has a known upper bound. More precisely:

$$
J(\mathbf{K}) \leq J_{\rho(i)}(\mathbf{K}) \leq \frac{1}{1-\left(1-\frac{\mu_{1}}{\mu_{2}}\right)^{2 i}} J(\mathbf{K})
$$

Proof: Define the following functions:

$$
P_{i}(\boldsymbol{\alpha}):=\frac{H(\boldsymbol{\alpha})}{h(\boldsymbol{\alpha})} \times \frac{1-\left(1-\frac{h(\boldsymbol{\alpha})}{\mu_{2}}\right)^{2 i}}{1-\left(1-\frac{\mu_{1}}{\mu_{2}}\right)^{2 i}}, \quad i=1,2, \ldots
$$

It is straightforward to show that $\operatorname{deg}\left(P_{i}\right)=\rho(i)$, and that $P_{i}(\boldsymbol{\alpha})$ is a polynomial (as opposed to a nonpolynomial rational function) which is positive definite 
over $\mathcal{D}$. Moreover, it can be concluded from (17) that:

$$
\frac{1-\left(1-\frac{h(\boldsymbol{\alpha})}{\mu_{2}}\right)^{2 i}}{1-\left(1-\frac{\mu_{1}}{\mu_{2}}\right)^{2 i}} \geq 1, \quad \forall \boldsymbol{\alpha} \in \mathcal{D}
$$

Thus, since $G(\boldsymbol{\alpha})=\frac{H(\boldsymbol{\alpha})}{h(\boldsymbol{\alpha})}$ satisfies the equation (8), one can write:

$$
\begin{aligned}
& (A(\boldsymbol{\alpha})+B(\boldsymbol{\alpha}) K(\boldsymbol{\alpha}) C(\boldsymbol{\alpha}))^{T} P_{i}(\boldsymbol{\alpha})(A(\boldsymbol{\alpha}) \\
& +B(\boldsymbol{\alpha}) K(\boldsymbol{\alpha}) C(\boldsymbol{\alpha}))-P_{i}(\boldsymbol{\alpha})+Q(\boldsymbol{\alpha}) \\
& +C(\boldsymbol{\alpha})^{T} K(\boldsymbol{\alpha})^{T} R K(\boldsymbol{\alpha}) C(\boldsymbol{\alpha}) \\
& =\left(Q(\boldsymbol{\alpha})+C(\boldsymbol{\alpha})^{T} K(\boldsymbol{\alpha})^{T} R K(\boldsymbol{\alpha}) C(\boldsymbol{\alpha})\right) \\
& \times\left(1-\frac{1-\left(1-\frac{h(\boldsymbol{\alpha})}{\mu_{2}}\right)^{2 i}}{1-\left(1-\frac{\mu_{1}}{\mu_{2}}\right)^{2 i}}\right) \leq 0
\end{aligned}
$$

for all $\alpha \in \mathcal{D}$. By applying the Schur complement formula to the above inequality, it can be verified that the inequality (15) is satisfied for $P(\boldsymbol{\alpha})=P_{i}(\boldsymbol{\alpha})$. Therefore, it can be deduced from Definition 5 that:

$$
J_{\rho(i)}(\mathbf{K}) \leq \operatorname{trace}\left(X_{0} \int_{\mathcal{D}} P_{i}(\boldsymbol{\alpha}) f(\boldsymbol{\alpha}) d \boldsymbol{\alpha}\right)
$$

On the other hand:

$$
1-\left(1-\frac{h(\boldsymbol{\alpha})}{\mu_{2}}\right)^{2 i} \leq 1-\left(1-\frac{\mu_{2}}{\mu_{2}}\right)^{2 i}=1, \quad \forall \boldsymbol{\alpha} \in \mathcal{D}
$$

The inequality (18) follows from the relations (7), (19), (22) and (23). Moreover, applying the squeezing theorem to the relation (18) yields that $\lim _{i \rightarrow+\infty} J_{\rho(i)}(\mathbf{K})=$ $J(\mathbf{K})$.

Remark 1 In order to investigate the tightness of the lower bound given in (18), consider the case when the Lyapunov function $\frac{H(\boldsymbol{\alpha})}{h(\boldsymbol{\alpha})}$ turns out to be a polynomial. In this case, $\mu_{1}$ and $\mu_{2}$ are equal (after cancelling common factors of $H(\boldsymbol{\alpha})$ and $h(\boldsymbol{\alpha})$, if any). Hence, it is concluded from(18) that $J(\mathbf{K})=J_{\rho(i)}(\mathbf{K}), \forall i>0$, which together with the monotone property of the sequence $\left\{J_{i}(\mathbf{K})\right\}_{1}^{\infty}$ yields that $J_{i}(\mathbf{K})$ is always equal to $J(\mathbf{K})$. This implies that the bound obtained is tight in the sense that the equality can be reached in the special case of polynomial Lyapunov functions.

Remark 2 The result of Theorem 2 can be interpreted as follows: consider an optimization problem aiming at minimizing the cost function (7) subject to the constraint (8) for a rational variable $G(\boldsymbol{\alpha})$ and a matrix polynomial $K(\boldsymbol{\alpha})$. The rational function $G(\boldsymbol{\alpha})$ can be replaced by a polynomial variable $P(\boldsymbol{\alpha})$, and consequently (8) can be substituted by (15). This substitution has the following properties: (i) the solution of the optimization problem subject to the latter constraint converges from above to that subject to the former constraint as the degree of $P(\boldsymbol{\alpha})$ increases; (ii) this convergence is more or less exponential. Notice that property (i) could have been deduced from Bliman (2004), if the constraint (8) were not an equality (note that although the constraint of the optimization problem is an inequality, the infimum is reached once the inequality turns to an equality).

Since the objective function $J(\mathbf{K})$ to be minimized in this work involves an unknown rational function $G(\boldsymbol{\alpha})$ (as a result of Lemma 1), SOS techniques cannot directly be employed to solve the problem. Hence, the problem of minimizing the performance index $J_{i}(\mathbf{K})$ will be treated at this point for any fixed value of $i$, as an alternative strategy for addressing the optimal controller design (i.e., minimizing $J(\mathbf{K})$ ). It is worth mentioning that no matter how small or large the number $i$ is, $J_{i}(\mathbf{K})$ is an upper bound for $J(\mathbf{K})$. Minimization of $J_{i}(\mathbf{K})$ involves two variables $K(\boldsymbol{\alpha})$ and $P(\boldsymbol{\alpha})$ with the objective function (14) subject to the constraint (15). The main difficulty of this problem is that one of the block entries of the constraint matrix $\Gamma(\boldsymbol{\alpha})$ is a nonlinear function of the coefficients of the matrix polynomial $P(\boldsymbol{\alpha})$. To avoid this difficulty, the celebrated technique of introducing a slack variable (Cao and Lam, 2001; Lavaei and Aghdam, 2007b) can be exploited. This idea is used here to develop an iterative procedure in order to find a nearoptimal controller $\mathbf{K}_{k}$ based on the initial controller $\mathbf{K}_{0}$, which requires solving a hierarchy of SOS optimization problems.

\section{Algorithm 1:}

Step 1) Find a Lyapunov polynomial $P_{0}(\boldsymbol{\alpha})$ for which there exist a positive scalar $\varepsilon$ and SOS matrix polynomials $Q_{0}(\boldsymbol{\alpha}), \ldots, Q_{\eta}(\boldsymbol{\alpha})$ so that the equation (24) holds for all $\boldsymbol{\alpha} \in \Re^{\mu}$.

Step 2) Set $\bar{P}(\boldsymbol{\alpha})=P_{0}(\boldsymbol{\alpha})$.

Step 3) Choose a natural number $i$ greater than or equal to $\operatorname{deg}\left(P_{0}\right)$ (see Remark 6).

Step 4) Solve an LMI optimization problem with the variables:

- a positive scalar $\varepsilon$;

- a matrix polynomial $K(\boldsymbol{\alpha})$ of degree $n_{0}$;

- SOS matrix Polynomials $Q_{0}(\boldsymbol{\alpha}), \ldots, Q_{\eta}(\boldsymbol{\alpha})$;

- a matrix polynomial $P(\boldsymbol{\alpha})$ of an arbitrary degree $i$; to minimize the objective function:

$$
\operatorname{trace}\left(X_{0} \int_{\mathcal{D}} P(\boldsymbol{\alpha}) f(\boldsymbol{\alpha}) d \boldsymbol{\alpha}\right)
$$

under the SOS constraint:

$$
\bar{\Gamma}(\boldsymbol{\alpha})=-Q_{0}(\boldsymbol{\alpha})-\sum_{j=1}^{\eta} q_{j}(\boldsymbol{\alpha}) Q_{j}(\boldsymbol{\alpha})-\varepsilon I
$$




$$
\left[\begin{array}{cc}
P_{0}(\boldsymbol{\alpha}) & \left(A(\boldsymbol{\alpha})+B(\boldsymbol{\alpha}) K_{0}(\boldsymbol{\alpha}) C(\boldsymbol{\alpha})\right)^{T} P_{0}(\boldsymbol{\alpha}) \\
P_{0}(\boldsymbol{\alpha})\left(A(\boldsymbol{\alpha})+B(\boldsymbol{\alpha}) K_{0}(\boldsymbol{\alpha}) C(\boldsymbol{\alpha})\right) & P_{0}(\boldsymbol{\alpha})
\end{array}\right]=Q_{0}(\boldsymbol{\alpha})+\sum_{j=1}^{\eta} q_{j}(\boldsymbol{\alpha}) Q_{j}(\boldsymbol{\alpha})+\varepsilon I
$$

where:

$$
\bar{\Gamma}(\boldsymbol{\alpha}):=\left[\begin{array}{ccc}
\Gamma_{11}(\boldsymbol{\alpha}) & * & * \\
\Gamma_{21}(\boldsymbol{\alpha}) & \bar{\Gamma}_{22}(\boldsymbol{\alpha}) & * \\
\Gamma_{31}(\boldsymbol{\alpha}) & \Gamma_{32}(\boldsymbol{\alpha}) & -I
\end{array}\right]
$$

with:

$$
\begin{aligned}
\bar{\Gamma}_{22}(\boldsymbol{\alpha}) & =-P(\boldsymbol{\alpha})-\bar{P}(\boldsymbol{\alpha}) B(\boldsymbol{\alpha}) R^{-1} B(\boldsymbol{\alpha})^{T} P(\boldsymbol{\alpha}) \\
& -P(\boldsymbol{\alpha}) B(\boldsymbol{\alpha}) R^{-1} B(\boldsymbol{\alpha})^{T} \bar{P}(\boldsymbol{\alpha}) \\
& +\bar{P}(\boldsymbol{\alpha}) B(\boldsymbol{\alpha}) R^{-1} B(\boldsymbol{\alpha})^{T} \bar{P}(\boldsymbol{\alpha})
\end{aligned}
$$

Step 5) If $\|P(\boldsymbol{\alpha})-\bar{P}(\boldsymbol{\alpha})\|_{e} \leq \delta$, where $\delta$ is a prescribed error margin (which is chosen in line with the design specifications), go to Step 7 .

Step 6) Set $\bar{P}(\boldsymbol{\alpha})$ to $P(\boldsymbol{\alpha})$, where $P(\boldsymbol{\alpha})$ is obtained in Step 4. Go to Step 4.

Step 7) The cost function $J_{i}\left(\mathbf{K}_{k}\right)$ is sufficiently close to a (local) solution. The polynomial $K(\boldsymbol{\alpha})$ obtained corresponds to a static near-optimal robust controller.

Remark 3 The reason why a function $P_{0}(\boldsymbol{\alpha})$ satisfying the condition in Step 1 of the above algorithm exists is thoroughly explained in Lavaei and Aghdam (2008).

Remark 4 Even though the objective function (25) is associated with the integral operator, it can be written as a linear combination of the variables being sought. Hence, after taking the integral of the prescribed monomials over the region $\mathcal{D}$, a linear expression in the conventional form will be attained as the objective function of the underlying optimization problem.

Remark 5 Algorithm 1 solves the optimization problem given in Step 4 recursively; therefore, the computational complexity of the algorithm is directly related to this optimization. It can be easily proved that the equality constraint of this optimization is in the form of linear SOS. Moreover, the objective function of this optimization problem is linear in terms of the coefficients of $P(\boldsymbol{\alpha})$. As a result, Step 4 of this algorithm can be easily performed by using the available software tools (Lofberg, 2004; Prajna, Papachristodoulou, Seiler and Parrilo, 2004). It is worth mentioning that the degrees of the polynomials involved in this optimization (i.e. $\left.Q_{0}(\boldsymbol{\alpha}), \ldots, Q_{\eta}(\boldsymbol{\alpha})\right)$ should be chosen beforehand. In this regard, a fruitful discussion is provided in Lavaei and Aghdam (2008) to obtain upper bounds on the degrees of the relevant polynomials.

Remark 6 Since $J_{i}(\mathbf{K})$ is minimized in Algorithm 1 instead of $J(\mathbf{K})$, it may turn out that choosing a very small value for $i$ improves the control performance insignificantly (or in an unlikely circumstance, the performance of the resultant closed-loop system may even degrade). To overcome this drawback, one can pursue the following strategy:

- Calculate the initial performance $J\left(\mathbf{K}_{0}\right)$ numerically.

- Find a proper value of $i$ for which $J_{i}\left(\mathbf{K}_{0}\right)$ is reasonably close to $J\left(\mathbf{K}_{0}\right)$. This can be achieved by using the bound given in Theorem 2 and the method discussed in Lavaei and Aghdam (2008) for finding the required parameters (e.g. $\mu_{1}$ and $\left.\mu_{2}\right)$.

- Design a controller $\mathbf{K}$ using Algorithm 1, and then evaluate the resultant closed-loop performance $J_{i}(\mathbf{K})$ numerically.

- If the performance $J_{i}(\mathbf{K})$ is close to $J\left(\mathbf{K}_{0}\right)$, there might be two possibilities: (i) the difference between $J_{i}\left(\mathbf{K}_{0}\right)$ and $J\left(\mathbf{K}_{0}\right)$ has not been sufficiently small; (ii) the initial controller is itself near-optimal. To figure out which case is true, one can increase $i$ and redo the algorithm provided $J_{i}\left(\mathbf{K}_{0}\right)$ and $J\left(\mathbf{K}_{0}\right)$ are reasonably far apart.

It is worth mentioning that as shown later in the numerical example, typically Algorithm 1 leads to a near-optimal controller efficiently, even by considering a very small value for $i$ which results in a large deviation $J_{i}\left(\mathbf{K}_{0}\right)-$ $J\left(\mathbf{K}_{0}\right)$.

\section{$3.1 H_{\infty}$ optimal controller design}

Designing a guaranteed $H_{\infty}$ controller for parameterdependent systems can be accomplished in line with the results developed here. In fact, one can start from the KYP lemma (Dullerud and Paganini, 2005), instead of Lemma 1, and translate the technicalities of this paper accordingly for the KYP condition. This will eventually lead to an SOS formula, which is very similar to the one obtained here. This means that the procedures for guaranteed $\mathrm{H}_{2}$ and $H_{\infty}$ optimal controller designs are analogous. Due to the space restrictions, the counterpart of Algorithm 1 for $H_{\infty}$ controller design is not delineated here.

\subsection{Comparison with existing works}

Let the results of this work be compared with some existing works in two special cases of deterministic systems and uncertain polytopic systems. In the former case, the formulation can be significantly simplified in order to arrive at the results derived in Cao and Lam (2001). A more interesting case, namely the polytopic uncertainty, 
is investigated in Vesely (2005). The method developed therein relies on approximating the rational Lyapunov function by a first-order polynomial, which in turn may be extremely conservative. It can be observed that by making several relaxations in this particular case, the SOS procedure given here encompasses the algorithm obtained in Vesely (2005). Therefore, not only does the result attained here reflect the very general case of uncertainty, it also encompasses some of the existing works.

\section{Numerical example}

Let $\mathcal{S}$ be an uncertain fourth-order system with the state-space matrices $A=\alpha_{1} \alpha_{2}^{2} \tilde{A}, B=\alpha_{1} \tilde{B}+\alpha_{2}^{2} I_{4}$ and $C=\alpha_{2}^{2} \tilde{C}+\alpha_{1} I_{4}$, where

$$
\begin{aligned}
& \tilde{A}=\left[\begin{array}{cccc}
-0.6957 & 0.2674 & -0.0031 & -0.0257 \\
0.6457 & -0.3634 & 0.0019 & -0.1042 \\
0.1407 & 0.5206 & -0.8307 & -0.2681 \\
0.0797 & -0.0139 & 0.0197 & -0.9133
\end{array}\right], \\
& \tilde{B}=\left[\begin{array}{cccc}
0.4518 & -0.2859 & -0.0059 & 0.0861 \\
0.8478 & -0.6839 & -0.0039 & 0.0889 \\
0.5211 & -0.1206 & -0.2406 & -0.7968 \\
0.2219 & -0.1109 & -0.1109 & 0.2407
\end{array}\right], \\
& \tilde{C}=\left[\begin{array}{cccc}
-0.3790 & -0.2632 & 0.0073 & 0.0076 \\
0.4781 & 0.6674 & 0.0224 & -0.1912 \\
-0.5010 & -0.2988 & 0.3827 & 0.2004 \\
0.0076 & 0.4705 & 0.0983 & -0.3630
\end{array}\right]
\end{aligned}
$$

and where $\alpha_{1}$ and $\alpha_{2}$ are the uncertain variables belonging to the region $\mathcal{D}=\left\{\left(\alpha_{1}, \alpha_{2}\right) \mid \alpha_{1}^{2}+\alpha_{2}^{2}=1\right\}$. It can be verified that the system $\mathcal{S}$ is stable under the unity feedback controller (this can be deduced from Example 1 in Lavaei and Aghdam (2008), after some manipulations). Consider the cost function (4) with the parameters $X_{0}=I_{2}, Q(\boldsymbol{\alpha})=R=I_{2}, f(\boldsymbol{\alpha})=1, \forall \boldsymbol{\alpha} \in \mathcal{D}$. Algorithm 1 is utilized to improve the performance of the unity feedback controller, by setting the degree of $P(\boldsymbol{\alpha})$ to 2 . The results obtained are summarized in Table 1. One can observe that a noticeable improvement is achieved after only one iteration, and that the algorithm converges in 10 iterations, resulting in \%57 improvement in the control performance. The optimal controller obtained is as follows:

$$
u[\kappa]=\left[\begin{array}{cccc}
0.1341 & -0.0332 & 0.0048 & 0.0361 \\
-0.0884 & 0.0323 & 0.0341 & 0.0096 \\
-0.0739 & -0.0421 & 0.1458 & 0.0837 \\
0.0060 & -0.0338 & -0.0273 & 0.1787
\end{array}\right] y[\kappa]
$$

On the other hand, one can employ a grid technique to calculate the performance indices numerically as follows:

$$
\begin{aligned}
& J\left(\mathbf{K}_{0}\right)=16.0996, \quad J_{2}\left(\mathbf{K}_{0}\right)=20.5719, \\
& J\left(\mathbf{K}_{k}\right)=8.4898, \quad J_{2}\left(\mathbf{K}_{k}\right)=8.8265
\end{aligned}
$$

These values point out that although the approximation of the rational Lyapunov function with a polynomial of degree 2 is not acceptable for the initial controller, the algorithm works remarkably well. Note that a static controller with a constant gain has been designed here, but in general Algorithm 1 can be used to design any parameter-dependent gain in the form of a polynomial with a prescribed degree.

\section{Conclusions}

This paper deals with the high-performance robust control synthesis for uncertain discrete-time LTI systems whose state-space matrices are polynomial functions of the uncertainty variables. Given a robustly statibilizing parameter-dependent static controller, it is aimed to tune the gain of this controller so that a near-optimal controller is obtained with respect to a prescribed quadratic cost function. To this end, it is shown that the underlying problem can be recast as a sum-of-squares (SOS) optimization. An illustrative example is provided to shed light on the main contribution of the paper.

\section{References}

Bakhilina, I. M., \& Stepanov, S. A. (2001). Design of robust linear controllers under parametric uncertainty of the object model. Automat. Remote Contr. $62(1), 101-113$.

Bliman, P. A. (2004). An existence result for polynomial solutions of parameter-dependent LMIs. Sys. Contr. Lett. 51(3-4), 165-169.

Cao, Y. Y., \& Lam, J. (2001). A computational method for simultaneous LQ optimal control design via piecewise constant output feedback. IEEE Trans. Sys. Man Cyber. 31(5), 836-842.

Chesi, G., Garulli, A., Tesi, A., \& Vicino, A. (2005). Polynomially parameter-dependent Lyapunov functions for robust stability of polytopic systems: an LMI approach. IEEE Trans. Automat. Contr. 50(3), 365-370.

Dullerud, G. E., \& Paganini, F. (2005). A course in robust control theory: A convex approach. Texts in Applied Mathematics, Springer.

Feron, E., Apkarian, P., \& Gahinet, P. (1996). Analysis and synthesis of robust control systems via parameter-dependent Lyapunov functions. IEEE Trans. Automat. Contr. 41(7), 1041-1046.

Foo, Y. K., \& Soh, Y. C. (1993). Robust stability bound for systems with structured and unstructured perturbations. Automat. Remote Contr. 38(7), 11501154 . 
Table 1

The results obtained by using Algorithm 1

\begin{tabular}{|l|l|l|l|l|l|l|l|l|l|l|l|}
\hline & $j=0$ & $j=1$ & $j=2$ & $j=3$ & $j=4$ & $j=5$ & $j=6$ & $j=7$ & $j=8$ & $j=9$ & $j=10$ \\
\hline static gain of degree 0 & 20.5719 & 13.1489 & 10.4558 & 9.4193 & 9.0164 & 8.8760 & 8.8407 & 8.8312 & 8.8277 & 8.8268 & 8.8265 \\
\hline
\end{tabular}

Gao, H., Lam, J., Xie, L., \& Wang, C. (2005). New approach to mixed $H_{2} / H_{\infty}$ filtering for polytopic discrete-time systems. IEEE Trans. Signal Processing. 53(8), 3183-3192.

Henrion, D., \& Lasserre, J. B. (2006). Convergent relaxations of polynomial matrix inequalities and static output feedback. IEEE Trans. Automat. Contr. 51(2), 192-202.

Goncalves, E. N., Palhares, R. M., \& Takahashi, R. H. C. (2005). Improved optimisation approach to the robust $\mathrm{H}_{2} / \mathrm{H}_{\infty}$ control problem for linear systems. Proc. IEE Contr. Theo. Appl. 152(2), 171-176.

Jibetean, D., \& Klerk, E. D. (2006). Global optimization of rational functions: a semidefinite programming approach. Mathematical Programming. 106(1), 93109.

Kau, S., Liu, Y., Hong, L., Lee, C., Fang, C., \& Lee, L. (2005). A new LMI condition for robust stability of discrete-time uncertain systems. Sys. Contr. Lett. 54(12), 1195-1203.

Lavaei, J., \& Aghdam, A. G. (2006a). Robust stability of discrete-time systems using sum-of-squares matrix polynomials. Proc. 2006 American Contr. Conf. (pp. 3828-3830).

Lavaei, J., \& Aghdam, A. G. (2006b). A necessary and sufficient condition for robust stability of LTI discrete-time systems using sum-of-squares matrix polynomials. Proc. of 45 th IEEE Conf. on Decision and Contr. (pp. 2924-2930).

Lavaei, J., \& Aghdam, A. G. (2007a). Optimal periodic feedback design for continuous-time LTI systems with constrained control structure. Int. J. Contr. 80(2), 220-230.

Lavaei, J., \& Aghdam, A. G. (2007b). Simultaneous LQ control of a set of LTI systems using constrained generalized sampled-data hold functions. Automatica. 43(2), 274-280.

Lavaei, J., \& Aghdam, A. G. (2008). Robust stability of LTI systems over semi-algebraic sets using sum-ofsquares matrix polynomials. IEEE Trans. Automat. Contr. 53(1), 417-423.

Lin, C., Wang, Q. G., \& Lee, T. H. (2006). A less conservative robust stability test for linear uncertain time-delay systems. IEEE Trans. Automat. Contr. 51(1), 87-91.

Lofberg, J. (2004). A toolbox for modeling and optimization in MATLAB. Proc. of the CACSD conf. (available online at http://control.ee.ethz.ch/ joloef/yalmip.php).

Oliveira, M. C. de, \& Geromel, J. C. (2005). A class of robust stability conditions where linear parameter dependence of the Lyapunov function is a necessary condition for arbitrary parameter dependence. Sys.
Contr. Lett. 54(11), 1131-1134.

Oliveira, P. J. de, Oliveira, R. C. L. F., Leite, V. J. S., Montagner, V. F., \& Peres, P. L. D. (2004). $H_{2}$ guaranteed cost computation by means of parameter dependent Lyapunov functions. Int. J. Sys. Sci. $35(5), 305-315$.

Oliveira, R. C. L. F., \& Peres, P. L. D. (2006). LMI conditions for robust stability analysis based on polynomially parameter-dependent Lyapunov functions. Sys. Contr. Lett. 55(1), 52-61.

Prajna, S., Papachristodoulou, A., Seiler, P., \& Parrilo, P. A. (2004). SOSTOOLS sum of squares optimization toolbox for MATLAB. Users guide (available online at http://www.cds.caltech.edu/sostools).

Scherer, C. W., \& Hol, C. W. J. (2006). Matrix sumof-squares relaxations for robust semi-definite programs. Mathematical Programming. 107(1-2), 189211.

Sekaj, I., \& Vesely, V. (2005). Robust output feedback controller design: genetic algorithm approach. IMA J. Math Contr. Info. 22(3), 257-265.

Shaked, U. (2003). An LPD approach to robust $H_{2}$ and $H_{\infty}$ static output-feedback design. IEEE Trans. Automat. Contr. 48(5), 866-872.

Vesely, V. (2005). Static output feedback robust controller design via LMI approach. J. Elec. Eng. 56(12), 3-8. 